\title{
Third International Workshop on the Web of Things (WoT 2012)
}

\author{
Simon Mayer \\ Inst. for Pervasive Computing \\ ETH Zürich, Switzerland \\ simon.mayer@inf.ethz.ch
}

\author{
Dominique Guinard \\ Evrythng Ltd. \\ Zürich, Switzerland \\ dom@evrythng.com
}

\author{
Erik Wilde \\ EMC Corporation \\ Pleasanton, CA, USA \\ dret@berkeley.edu
}

\begin{abstract}
Continuing the successful Web of Things workshop series, the goal of this workshop is to further explore the use of technologies and principles at the core of the Web to provide methods for a seamless integration of physical devices. In particular, our goal is to foster discussion on systems towards a real-time Web of Things and the discovery, search, and composition of services provided by Web-enabled things. This document contains all submissions to WoT 2012 that were accepted after a double-blind peer-reviewing process and cover current research in the fields of Web-based service discovery and semantic descriptions, case studies and toolkits for the Web of Things, and architectures that demonstrate the possibilities which arise when combining Web patterns and technologies with metadata and machine learning approaches to create smart environments.
\end{abstract}

\section{Categories and Subject Descriptors}

C.2.0 [Computer Communication Networks]: General

\section{General Terms}

Algorithms, Design, Languages, Standardization, Theory

\section{Keywords}

Web of Things, REST, HTTP, Web Architecture, Internet of Things

\section{INTRODUCTION}

The world of embedded devices has experienced radical changes over the past few years. Real-world objects, or "Smart Things", such as home appliances, industrial machines, and wireless sensor and actuator networks embed powerful computers which often are connected to the Internet. Chumby, Gumstix, Sun SPOTs, Ploggs, Nabaztag, and ioBridges as well as the proliferation of data aggregation platforms like pachube are just a few examples of the

Permission to make digital or hard copies of all or part of this work for personal or classroom use is granted without fee provided that copies are not made or distributed for profit or commercial advantage and that copies bear this notice and the full citation on the first page. To copy otherwise, to republish, to post on servers or to redistribute to lists, requires prior specific permission and/or a fee.

WoT 2012, June 2012; Newcastle, UK

Copyright 2012 ACM 978-1-4503-1603-3/12/06 ...\$10.00. rapid development of such connected embedded computers. Considering the recent progress in mobile communications (increased bandwidth for cell phone networks, as well as urban wireless broadband networks), Internet access will very likely become a commodity available on most real-world devices. According to the IP for Smart Objects (IPSO) Alliance, an increasing number of embedded devices will be supporting IP, which will enable many physical objects to be connected to the Internet. This convergence of physical computing devices (wireless sensor networks, mobile phones, embedded computers, etc.) and the Internet provides new design opportunities and challenges, as digital communication networks will soon not only contain information resources (images, text, etc.), but also real objects.

The Internet of Things (IoT) has become a well-known brand for a set of research issues in the pervasive and ubiquitous computing communities. However, the focus of this research theme has mostly been on establishing connectivity in a variety of challenging and constrained networking environments. Our hypothesis is that the Web of Things (WoT) is the next logical step in the ongoing evolution of how pervasive and ubiquitous computing have enabled new applications and provided new opportunities. The Web of Things takes the next step from establishing connectivity and thus the ability to communicate with Things, to a vision where Things become seamlessly integrated into the Web not just through Web-based user interfaces of specific applications, but by blending into the hypermedia information space created by the Web and its architectural principles.

\section{WORKSHOP OBJECTIVES}

The primary goal of the Web of Things 2012 workshop series is to take the wealth of research and applications already available in the the pervasive and ubiquitous computing communities, and to identify the issues that need to be addressed to make them available as part of the Web. Specifically, our goal is to highlight and focus on the difference between Web-based user interfaces ("having Web UIs for things"), and Web services ("immersing things into the Web"). Embracing the REST paradigm [2], interactions with things should work in the very same way as interactions with other resources on the Web, allowing developers to mix resources and their provided services in ways similar to the popular Web 2.0 mashups [3, 4]. One of the key goals of the workshop is to foster collaboration among researchers in pervasive and ubiquitous computing, and to encourage them to build loosely coupled architectures to allow the various systems and architectures devised by researchers and 
engineers around the world to cooperate seamlessly.

After laying the foundation of the Web of Things over the last three years, we observe that this approach and its underlying concepts are gaining substantial momentum. The fast growing webofthings.org community, and the recent calls for Web/Internet of Things projects launched by the European Community clearly demonstrate the growing interest in the concept. The increasing number of Web of Things-related scientific publications in the field of Pervasive Computing $[1,4,7,8,9]$ also confirms this fact. Furthermore, the recent developments in Embedded Computing and Wireless Sensor Networks [5, 6], along with the successful IP for Smart Object (IPSO) Alliance, clearly illustrate the need and benefits to be gained from making embedded devices part of the Internet. However, these trends illustrate only connectivity at the TCP/IP level without actually considering what goes on top, at the application level, where a standard protocol is lacking. The Web of Things workshop series targets exactly this gap and enables necessary discussions on the important issues and technical challenges of a global and scalable application layer for embedded devices. It furthermore allows researchers and practitioners to demonstrate innovative applications and use cases for the Web of Things, in particular to tackle the great challenges we face today in the fields of transportation, healthcare, elderly care, sustainability, and energy awareness.

\section{WORKSHOP TOPICS}

In general, the WoT workshops explore the use of technologies at the core of the Web such as Representational State Transfer (REST), syndication (e.g., Atom) and realtime Web technologies (e.g., Web Sockets, XMPP, Pubsubhubbub, etc.), load-balancing, etc. to provide access to ubiquitous computing services provided by smart things. They also aim at better understanding and tackling the challenges when building a seamless Web of Things, where devices can freely interact with each other, with human users, and software applications. The general scope and relevant topics for the workshop are described as follows:

- Discovery and look-up for things and their services on the Web

- Web-based things composition and physical mashups

- Real-time communication with physical objects (e.g., syndication, streaming, instant messaging, Web push)

- Human-things interaction models and paradigms

- Security, access control, and sharing of physical things on the Web

- Application of Web tools and techniques for the physical world (e.g., REST, HTML5, caching, cloud services, social networks)

- Applications, deployments and evaluations of Web of things systems

- Business opportunities for the Web of Things

- Use of semantic technologies (e.g., ontologies, microformats) in the context of the Web of Things

- Interaction models and paradigms between humans and Web-enabled things (e.g., mobile/tangible interfaces)

\section{WOT 2012 AND THE WOT WORKSHOP SERIES}

WoT 2012 continued the successful Web of Things workshop series at PerCom 2010 (Mannheim, Germany) and Pervasive 2011 (San Francisco, US), as well as the Urban-IoT 2010 workshop at the Internet of Things 2010 conference (Tokyo, Japan). This third iteration is thus already building on a solid community of participants and reviewers who contribute in core domains of the WoT (e.g., deployments, Web of Things kits and frameworks, Web of Things architectures) but still was able to extend to new fields around the context-aware WoT, e.g., to semantics and artificial intelligence. The best paper award this year was given to Michael Blackstock and Rodger Lea for their paper "WoTKit: A Lightweight Toolkit for the Web of Things", an appreciation of their continuous work to create a toolkit that fosters the creativity of WoT researchers and allows them to focus on the actual use-cases.

Just as in 2011, a hackathon was organized before the workshop. The motto of this year's hackaton was "When dumb things get an identity on the Web...". The dozen of participants hence focused on connecting everyday objects with IoT services (such as the EVRYTHNG API) by means of QR-codes and NFC tags. At the end of the day, two applications had emerged: "Coffee Trace", which enabled the tracking and crowd-sourced reviews of coffee beans and "FreezeMe", an application making it easy to prevent foodwaste by having your frozen food items warning you (e.g., through twitter) when they are coming close to their expiry date.

This year's workshop also included an open demonstration session, where participants could bring and demonstrate any projects or prototypes that they thought were relevant for the WoT community. In the end, six projects were demonstrated and greatly helped to foster discussions and interaction between participants. Furthermore, three commercial startups - openPicus, Koubachi, and EVRYTHNG - presented their companies during keynote-talks in-between the workshop's three technical sessions. The sessions themselves were structured around the topics of the "Context-aware and Semantic Web of Things", "Web of Things Enabling and Enablers", and "Web of Things Architectures and Mashups".

\section{ORGANIZERS}

This year's workshop was organized jointly by Simon Mayer (ETH Zürich), Dominique Guinard (Evrythng Ltd.), and Erik Wilde (EMC Corp.).

Simon Mayer is working as a PhD student and Research Assistant in the Internet of Things/Web of Things domain at the Institute for Pervasive Computing, ETH Zürich. His main research topics are aspects of integrating smart things into the Web including the deployment of such devices, their semantic description, and infrastructures that support human users and machines in finding and using the information and services they provide. He is also interested in facilitating the interaction with smart things for human end users and in empowering them to monitor and control their things and to create physical mashups. Simon graduated in Computer Science from ETH Zürich.

Dominique Guinard is the CTO of an Internet of Thingsrelated startup called Evrythng. He is also the co-founder of the Web of Things initiative and workshop series. During 
his $\mathrm{PhD}$ at ETH Zürich, he researched on the foundations of the Web of Things and especially focused on facilitating application development in the form of physical mashups. He was also a visiting researcher at the Auto-ID Labs of MIT, working on bringing global networks of tagged objects to the Web. Before this he worked 4 years as a research associate for SAP Research, building a service oriented architecture to enable real-world device integration into business software. Dominique had further experiences with several research institutions and companies in the Internet of Things domain such as with Lancaster University, Nokia research or Sun Microsytems. He graduated in Computer Science from the universities of Fribourg and Bern.

Erik Wilde works as an architect at EMC, and is responsible for the long-term move of EMC's content management products to a RESTful service-oriented architecture. Until mid-2011, Erik has worked as adjunct professor at the UC Berkeley School of Information. He has over 10 years of background in working in Web-related fields, ranging from $\mathrm{XML}$ and data modeling issues to questions of Web services and RESTful design principles. Erik graduated from TU Berlin in computer science in 1991, got his PhD from ETH Zürich in computer communications in 1997, and since then has worked at ICSI in Berkeley, ETH Zürich, and for the past four years at UC Berkeley's School of Information, where he teaches Web Architecture and XML technologies. Two of his main research themes in the past years have been how to make the Web location-aware, and how to design and implement loosely coupled RESTful services; both of these themes have very close ties to the "Web of Things". Apart from the "Web of Things" workshop series, he also organizes the Location and the Web (LocWeb) and Workshop for RESTful Design (WS-REST) workshop series.

\section{PROGRAM COMMITTEE}

The program committee of WoT 2012 has been assembled to thoroughly review all submissions to the workshop. Each submission was reviewed by up to five PC members in a double-blind fashion, which resulted in a fair and balanced reviewing process and helpful feedback for the authors. The program committee of WoT 2012 consists of:

- Rosa Alarcon, UC Berkeley, USA

- Benoit Christophe, Alcatel Lucent Bell Labs, France

- Christian Floerkemeier, Auto-ID Labs, MIT, USA

- Artem Katasonov, VTT Technical Research Center, Finland

- Gerd Kortuem, University of Lancaster, UK

- Matthias Kovatsch, ETH Zürich, Switzerland

- Marc Langheinrich, Universita della Svizzera Italiana (USI), Switzerland

- Rodger Lea, University of British Columbia, Canada

- Olivier Liechti, Haute Ecole d'Ingénierie et de Gestion du Canton de Vaud (HEIG-VD), Switzerland

- Marino Linaje, Universidad de Extremadura, Spain

- Diego Lopez de Ipina, Universidad de Deusto, Spain

- Friedemann Mattern, ETH Zürich, Switzerland

- Florian Michahelles, ETH Zürich, Switzerland

- Guido Moritz, Universität Rostock, Germany

- Claro Noda, Instituto Politécnico de Porto, Portugal

- Jacques Pasquier, Université de Fribourg, Switzerland

- Cesare Pautasso, Universita della Svizzera Italiana (USI), Switzerland
- Dave Raggett, World Wide Web Consortium, UK

- David Resseguie, Oak Ridge National Laboratory, USA

- Till Riedel, TecO Karlsruhe Institute of Technology, Germany

- Andreas Ruppen, Université de Fribourg, Switzerland

- Vlad Stirbu, Nokia Research, Finland

- Vlad Trifa, Evrythng Ltd., Switzerland

- Iñaki Vázquez, Symplio, Spain

\section{ACKNOWLEDGEMENTS}

We would like to extend our thanks to our colleagues who agreed to serve on the program committee and to all authors and participants of WoT 2012. Thank you for helping us make the 3rd International Workshop on the Web of Things happen!

\section{MORE INFORMATION}

For more information about the workshop as well as all papers presented at WoT 2012, we invite the reader to visit the workshop's official Web page on http://www . webofthings . org/wot/2012.

\section{REFERENCES}

[1] R. Dickerson, J. Lu, J. Lu, and K. Whitehouse. Stream Feeds - An Abstraction for the World Wide Sensor Web. In Proc. of the 1st International Conference on the Internet of Things (IoT 2008), Zürich, Switzerland, 2008.

[2] R. T. Fielding. Architectural Styles and the Design of Network-based Software Architectures. PhD thesis, University of California, Irvine, Irvine, California, 2000.

[3] D. Guinard, V. Trifa, T. Pham, and O. Liechti. Towards Physical Mashups in the Web of Things. In Proc. of the 6th International Conference on Networked Sensing Systems (INSS 2009), Pittsburgh, USA, 2009.

[4] D. Guinard, V. Trifa, and E. Wilde. A Resource Oriented Architecture for the Web of Things. In Proc. of the 2nd International Conference on the Internet of Things (IoT 2010), Tokyo, Japan, 2010.

[5] J. W. Hui and D. E. Culler. Extending IP to Low-Power, Wireless Personal Area Networks. Internet Computing, IEEE, 12(4):37-45, 2008.

[6] J. W. Hui and D. E. Culler. IP is dead, long live IP for wireless sensor networks. In Proc. of the 6th ACM Conference on Embedded Networked Sensor Systems (SenSys 2008), Raleigh, USA, 2008.

[7] D. Raggett. Towards a Web of Things. Invited Presentation at the 1st International Conference on the Internet of Things (IoT 2008), Zürich, Switzerland, 2008.

[8] V. Stirbu. Towards a RESTful Plug and Play Experience in the Web of Things. In Proc. of the 2nd IEEE International Conference on Semantic Computing (ICSC 2008), Santa Clara, USA, 2008.

[9] J. Vazquez and D. Lopez-de-Ipina. Social Devices: Autonomous Artifacts That Communicate on the Internet. In Proc. of the 1st International Conference on the Internet of Things (IoT 2008), Zürich, Switzerland, 2008. 